\title{
Zonal Wind Calculations from Mars Global Surveyor Accelerometer and Rate Data
}

\author{
Darren T. Baird* \\ Jet Propulsion Laboratory, California Institute of Technology, Pasadena, California 91109-8099 \\ Robert Tolson $\ddagger$ \\ North Carolina State University, Hampton, Virginia 23666 \\ and \\ Stephen Bougher \pm and Brian Steers $\stackrel{\S}{ }$ \\ University of Michigan, Ann Arbor, Michigan 48109-2143
}

\section{DOI: $\underline{10.2514 / 1.28588}$}

\begin{abstract}
The Mars Global Surveyor spacecraft was initially placed into a high-eccentricity, nearly polar orbit about Mars with a 45-h period. To accomplish the science objectives of the mission, a 2-h circular orbit was required. Using a method known as aerobraking, numerous passes through the upper atmosphere slowed the spacecraft, thereby reducing the orbital period and eccentricity. To successfully perform aerobraking, the spacecraft was designed to be longitudinally, aerodynamically stable in pitch and yaw. Because the orbit was nearly polar, the yaw orientation of the spacecraft was sensitive to disturbances caused by the zonal components of wind (east to west or west to east) acting on the spacecraft at aerobraking altitudes. Zonal wind velocities were computed by equating the aerodynamic and inertia-related torques acting on the spacecraft. Comparisons of calculated zonal winds with those computed from the Mars thermospheric general-circulation model are discussed.
\end{abstract}

\section{Nomenclature}

$A \quad=$ spacecraft reference area

$B \quad=$ sensitivity matrix

$C_{d}=$ coefficient of drag

$C M=$ center of mass

$C m_{x}=$ yawing moment coefficient

$C m_{y}=$ pitching moment coefficient

$\mathrm{Cm}_{z}=$ rolling moment coefficient

$C_{x}=X$-direction force coefficient

$C_{y} \quad=\quad Y$-direction force coefficient

$C_{z}=Z$-direction force coefficient

$I \quad=$ moment-of-inertia matrix for single body

$J \quad=$ moment-of-inertia matrix for reaction wheels

$L=$ spacecraft reference length

$\bar{L} \quad=$ spacecraft angular momentum

$L_{s} \quad=$ solar longitude, ${ }^{\circ}$

$l=$ direction cosine with respect to the $x$ axis

$M \quad=$ spacecraft mass

$m \quad=$ direction cosine with respect to the $y$ axis

$\bar{N} \quad=$ external torques, $\mathrm{N} \cdot \mathrm{m}$

$n \quad=$ direction cosine with respect to the $z$ axis

$P_{x x x}=$ periapsis of orbit $x x x$

$r \quad=$ spacecraft-Mars distance

$\bar{r} \quad=$ spacecraft-centered position vector

Presented as Paper 6390 at the 2006 AIAA/AAS Astrodynamics Specialist Conference and Exhibit, Keystone, CO, 21-24 August 2006; received 30 October 2006; accepted for publication 10 May 2007. Copyright (C) 2007 by the American Institute of Aeronautics and Astronautics, Inc. The U.S. Government has a royalty-free license to exercise all rights under the copyright claimed herein for Governmental purposes. All other rights are reserved by the copyright owner. Copies of this paper may be made for personal or internal use, on condition that the copier pay the $\$ 10.00$ per-copy fee to the Copyright Clearance Center, Inc., 222 Rosewood Drive, Danvers, MA 01923; include the code 0022-4650/07 \$10.00 in correspondence with the CCC.

*Member of Engineering Staff, Guidance, Navigation and Control Section, Mail Stop 301-276. Lifetime Member AIAA.

${ }^{\dagger}$ Professor, Center for Planetary Atmospheric and Flight Sciences, 100 Exploration Way. Associate Fellow AIAA.

†Professor, Space Physics Research Laboratory, 2455 Hayward Avenue.

${ }^{\S}$ Student, Aerospace Engineering Department, 2455 Hayward Avenue. Student Member AIAA.

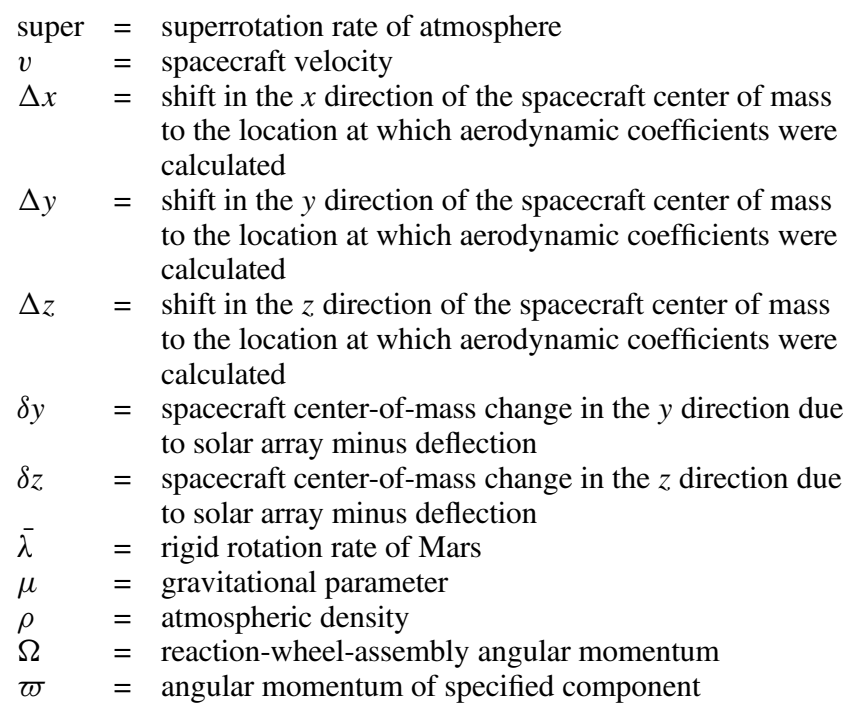

Subscripts

$\mathrm{B} / \mathrm{S}=$ bus/solar array plus $(+y$ axis $)$ combination

$\mathrm{gg} \quad=$ gravity gradient

$\mathrm{SAM}=$ solar array minus $(-y$ axis $)$

$\mathrm{S} / \mathrm{C}=$ spacecraft

\section{Introduction}

ATER a 10-month cruise from Earth, Mars Global Surveyor A (MGS) was placed into a highly elliptical, nearly polar, orbit about Mars. MGS was the first interplanetary probe for which mission success was dependent on the safe, timely, and effective use of aerobraking. The goal of aerobraking was to reduce the period of the nearly polar orbit from 45 to $2 \mathrm{~h}$ without relying on propulsive burns. Aerobraking was broken into two phases and used more than 1200 controlled passes through the upper atmosphere to gradually slow the spacecraft, thereby reducing the orbital period and eccentricity [1].

During each aerobraking pass, the solar arrays were swept aft approximately $30 \mathrm{deg}$, which shifted the center of pressure aft of the center of mass of the spacecraft. This configuration, shown in Fig. 1 , 


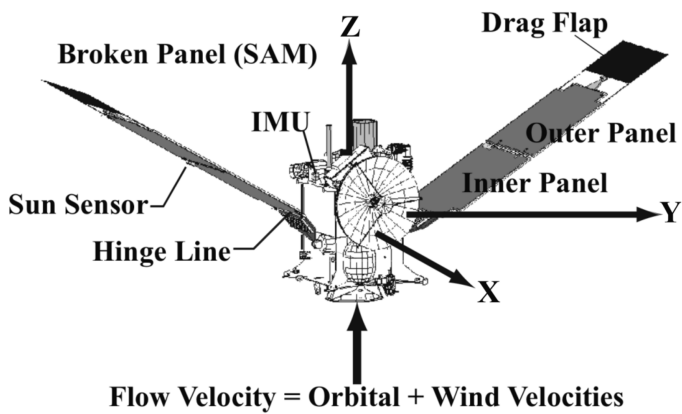

Fig. 1 MGS aerobraking schematic.

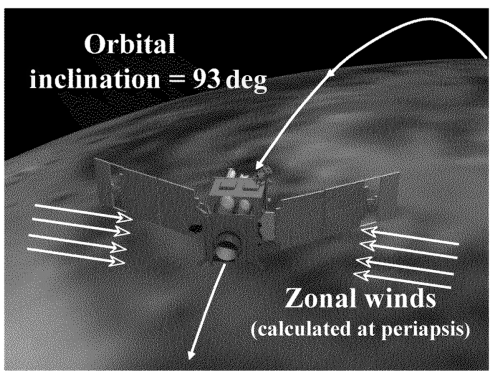

Fig. 2 Zonal wind components acting on MGS.

provided longitudinal aerodynamic stability in pitch and yaw throughout each aerobraking pass. Because the orbit was nearly polar, the yaw orientation of the spacecraft was sensitive to disturbances caused by zonal components of wind (east to west or west to east) acting on the spacecraft at aerobraking altitudes, as shown in Fig. 2. Figure 1 shows that the normal flow is along the $z$ axis, but preflight attitude control simulations indicated that during the aerobraking passes, the relative winds could deviate from the $z$ axis by as much as $15 \mathrm{deg}$, which could affect the zonal wind calculations by as much as $70 \mathrm{~m} / \mathrm{s}$.

As will be shown, accelerometers, rate gyros, and reaction wheels onboard MGS can serve as instruments to calculate these winds. Accelerometer data are first used to calculate the density profiles throughout each atmospheric pass. The reaction wheel data are used to calculate the effects of onboard momentum sources. Euler's equations of motion are then used to relate the aerodynamic and gravity gradient torques to the attitude motion of the spacecraft. By solving these equations, it is possible to determine what torques the spacecraft experiences due to local winds and, therefore, to calculate the components of wind acting on the vehicle.

\section{Euler's Equations of Motion}

To determine zonal winds, accelerometer data are first used to calculate the density of the atmosphere for the duration of each aerobraking pass using Eq. (1) $[2,3]$, where $M$ is the spacecraft mass, $v$ is the spacecraft velocity, $C_{d}$ is the drag coefficient, and $A$ is the spacecraft cross-sectional area in the aerobraking configuration.

$$
\rho=\left[2 M / V^{2} C_{d} A\right] \dot{v}
$$

For a rigid body, attitude motion is represented by Euler's equations, as shown in Eq. (2). The total angular momentum of the spacecraft is represented by $\bar{L}$, with contributions from the solar array minus (SAM), bus/solar array plus (bus/SAP) assembly, and the reaction wheels, and $\bar{N}$ represents the externally generated torques.

$$
\frac{\mathrm{d} \bar{L}}{\mathrm{~d} t}=\bar{N}
$$

Because Euler's equations of motion relate external torques to the time rate of change of angular momentum of the spacecraft, the torques acting upon the spacecraft by zonal winds can be calculated if all other external torques and the time rate of change of angular momentum can be determined. These external torques applied to the spacecraft include thruster firings, gravity gradient, and aerodynamics, whereas desaturation of the reaction wheels and the deflection of the SAM generate internal torques. These torques are calculated using spacecraft telemetry from the corresponding aerobraking pass.

\section{Equations of Motion for Two Rigid Bodies}

Because of the broken solar panel, MGS is modeled as two rigid bodies connected by a nonlinear torsional spring. The two bodies are the SAM and bus/SAP combination. Euler's equations can be written independently for each body with respect to an inertial frame, as shown in Eq. (3).

$$
\frac{\mathrm{d} \bar{L}}{\mathrm{~d} t}=\bar{N}=\frac{\mathrm{d}(I \bar{\omega})}{\mathrm{d} t}=I \dot{\omega}+\dot{I} \omega
$$

Calculating the time rate of change of the moments of inertia is difficult. However, an analysis was performed assuming a SAM deflection of $5 \mathrm{deg}$, a conservative value compared with the average measured inbound and outbound deflections $(-0.6$ to $+2.1 \mathrm{deg})$ described at the end of the Effects of Solar Array Minus Deflection section. A SAM deflection of $5 \mathrm{deg}$ corresponds to a maximum angular velocity of $0.0819 \mathrm{rad} / \mathrm{s}$. This analysis demonstrated that the last term of Eq. (3) is negligible for the $y$ and $z$ axes. More important, this term is zero for the $x$ axis, and because the zonal winds cause torques primarily about the $x$ axis, the last term of Eq. (3) was disregarded. Rotating body-fixed systems were chosen about the center of mass of the SAM and bus/SAP combination so that the moments of inertia of each body remain constant. When adding the angular momentum terms, a common basis must be referenced, which was the spacecraft center of mass with no SAM deflection. Therefore, Euler's equation for the two-body system is represented by Eq. (). .

$$
\begin{gathered}
I_{\mathrm{B} / \mathrm{S}} \dot{\varpi}_{\mathrm{B} / \mathrm{S}}+\varpi_{\mathrm{B} / \mathrm{S}} \times I_{\mathrm{B} / \mathrm{s}} \varpi_{\mathrm{B} / \mathrm{S}}+J \dot{\Omega}+\varpi_{\mathrm{B} / \mathrm{S}} \times J \Omega+I_{\mathrm{SAM}} \dot{\varpi}_{\mathrm{SAM}} \\
+\varpi_{\mathrm{SAM}} \times I_{\mathrm{SAM}} \varpi_{\mathrm{SAM}}-\bar{N}_{\mathrm{gg}}=0.5 \rho \bar{V}_{\mathrm{S} / \mathrm{C}}^{2} A L C_{m_{x}}
\end{gathered}
$$

The known terms (i.e., those obtained from attitude control system data) that do not depend on zonal winds are moved to the left-hand side (LHS) of Eq. (4) to yield the equation to be evaluated. The known terms are evaluated using the two body-fixed coordinate systems, one centered at the center of mass of the bus/SAP assembly and the other centered at the SAM center of mass. $C_{m_{x}}$ is the only term that significantly depends on zonal winds, and Fig. $\underline{3}$ shows $C_{m_{x}}$ as a function of yaw angle for panel deflections of $0,10, \overline{1} 7$, and $20 \mathrm{deg}$ in free molecular flow.

Figure $\underline{3}$ shows that the panel deflection causes a shift in $C_{m_{x}}$ such that a 10-deg panel deflection produces about $5 \mathrm{deg}$ of displacement in the equilibrium yaw angle. Also, $C_{m_{x}}$ varies linearly with the $y$ component of wind for a given dynamic pressure. Zonal winds cause a change in yaw angle, and $C_{m_{x}}$ must be calculated to determine zonal winds.

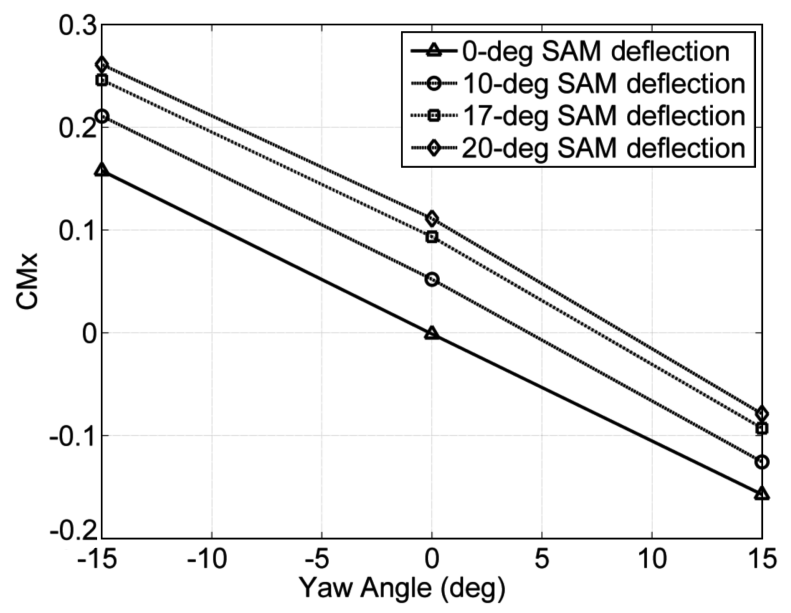

Fig. $3 C_{m_{x}}$ as a function of relative wind for different panel deflections. 
The attitude control system of MGS consists of rate gyros, reaction wheels, thrusters, and sun sensors. The angular velocity of the bus/ SAP combination, $\varpi_{\mathrm{B} / \mathrm{S}}$, is measured by the bus-fixed gyros, and these data are recorded at 1-s intervals throughout each aerobraking pass. Using a double-sided difference, the time derivatives of $\varpi_{\mathrm{B} / \mathrm{S}}$ are calculated. The angular velocity of the spacecraft near P52 is shown in Fig. 4.

The low-frequency variations in Fig. 4 are due to aerodynamic moments and have a characteristic time of approximately $50 \mathrm{~s}$. Also, Fig. 4 shows a 6-s oscillation $(0.15 \mathrm{~Hz})$ caused by the SAM deflection throughout the aerobraking pass. As the SAM oscillates, the bus must counterbalance the motion to preserve angular momentum if external torques are ignored. This 6-s oscillation is not of interest in the determination of zonal winds, and all data are filtered using a lowpass two-direction filter.

Panel position data are calculated at 1-s intervals throughout each aerobraking pass [4]. Angular velocities of the SAM are calculated using a double-sided difference of the filtered panel position, and angular accelerations are similarly calculated using a double-sided difference of the angular velocities.

Referring to Fig. 4, about $10 \mathrm{~s}$ before periapsis there is a change in angular velocity, shown by a sudden change in the slope in the $y$-axis angular momentum. This angular acceleration is due to a reaction wheel biasing event, which usually occurs near periapsis. Reaction wheels are not used for attitude control during aerobraking, because they cannot produce enough torque to overcome the moments produced by the aerodynamic forces, and so their speeds are held nearly constant. However, to minimize attitude control fuel usage, reaction wheel biasing events are performed near periapsis, in which aerodynamic torques easily balance the reaction wheel torques due to desaturation. For purposes of this method, a reaction wheel biasing event refers to the change in velocity from one nominal speed before periapsis to another nominal speed after periapsis. During each reaction wheel biasing event, the reaction wheel speeds are changed at a nearly constant acceleration.

Knowing that the three reaction wheels start desaturating at the same time, the maximum difference between the mean velocities of the reaction wheels before and after the biasing event is found, and a best-fit line is drawn through the points for which the reaction wheel velocity is changing. The time at which this line intercepts the mean velocity before the biasing event began is taken to be the bias-event start time. Figure 5 shows the original data, the linear fit to the data, and the lines of the mean reaction wheel speed before and after the biasing event, as represented by data from P52.

The mean reaction wheel speeds before the biasing event are used as the reaction wheel speeds for all times up to the biasing-event start time, and the mean reaction wheel speeds after the biasing event are considered as the reaction wheel speeds for all times after the biasing event.

The gravity gradient torques are calculated in Eq. (5) [5]. The values $1, m$, and $n$ are the direction cosines in the body system of the vector extending from the spacecraft to the center of Mars, and $r$ is

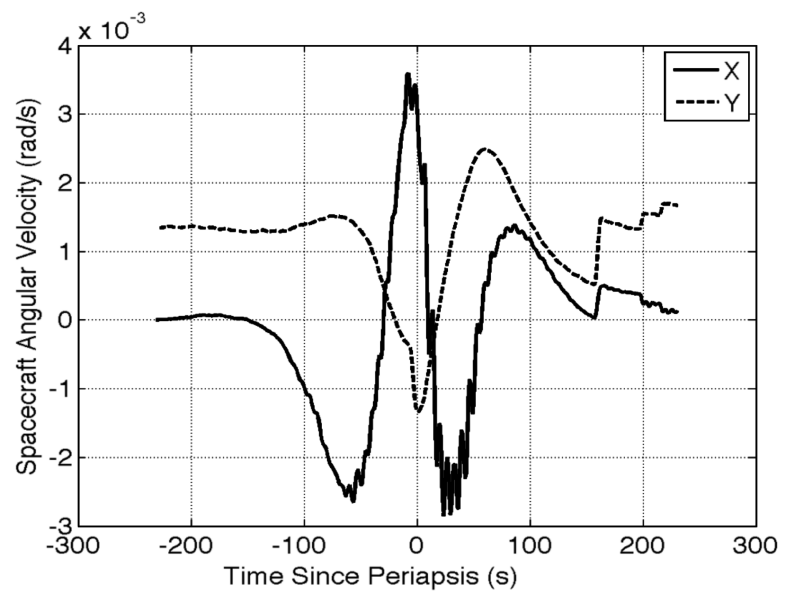

Fig. 4 Spacecraft angular velocity near P52.

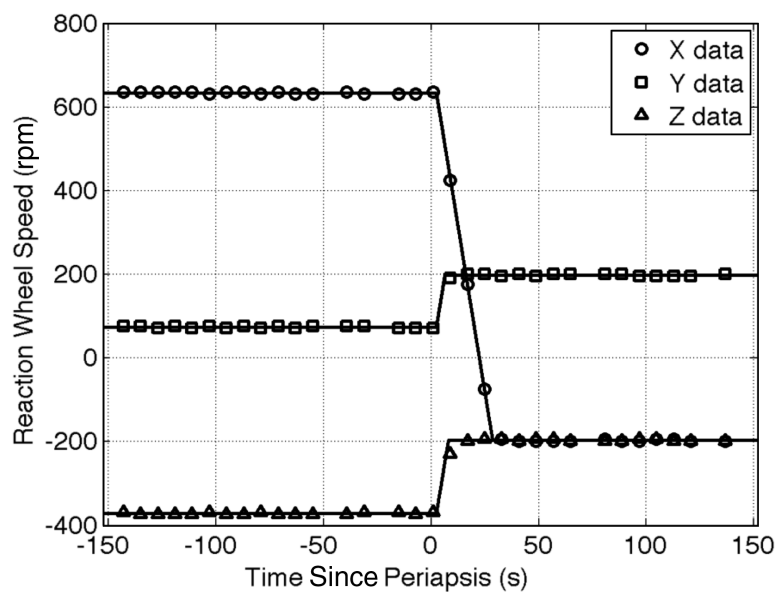

Fig. 5 Reaction wheel data for P52.

the distance from the spacecraft center of mass to the center of Mars.

$$
\begin{aligned}
& N_{X_{\mathrm{gg}}}=\left(3 \mu_{\mathrm{Mars}} / r^{3}\right)\left[m n\left(I_{z z}-I_{y y}\right)+n l I_{x y}-l m I_{x z}+\left(n^{2}-m^{2}\right) I_{y z}\right] \\
& N_{Y_{\mathrm{gg}}}=\left(3 \mu_{\mathrm{Mars}} / r^{3}\right)\left[n l\left(I_{x x}-I_{z z}\right)+\operatorname{lm} I_{y z}-m n I_{x y}+\left(l^{2}-n^{2}\right) I_{x z}\right] \\
& N_{Z_{\mathrm{gg}}}=\left(3 \mu_{\mathrm{Mars}} / r^{3}\right)\left[m l\left(I_{y y}-I_{x x}\right)+m n I_{x z}-n l I_{y z}+\left(m^{2}-l^{2}\right) I_{x y}\right]
\end{aligned}
$$

Gravity gradient torques are small compared with the other torques, but for completeness, they are retained to eliminate a source of error.

\section{Zonal Wind Terms}

As MGS passes through the atmosphere in a nearly polar orbit, three separate wind components act on the spacecraft. Vertical winds are generally on the order of $10 \mathrm{~m} / \mathrm{s}$ and are not expected to greatly affect the spacecraft attitude motion. Meridional winds, which are in the north/south direction, also do not greatly affect the spacecraft heading, because MGS is in a nearly polar orbit, and these winds contribute no more than a $100 \mathrm{~m} / \mathrm{s}$ velocity change along the orbital velocity vector and do not produce attitude changes. However, the zonal winds, which travel in the east/west direction (crosswinds with respect to the spacecraft), can significantly change the spacecraft heading, as illustrated in Fig. 2. For example, at P50, the spacecraft velocity was $4760 \mathrm{~m} / \mathrm{s}$ at $38.4^{\circ} \mathrm{N}$ latitude and at an altitude of $123.5 \mathrm{~km}$. Under those conditions, the inertial zonal winds at periapsis were calculated by taking the cross product of the rotation rate of the planet and the spacecraft position vector. The wind velocity is $171 \mathrm{~m} / \mathrm{s}$, which causes the heading to change by approximately $2 \mathrm{deg}$. It is normally assumed that the atmosphere is rigidly rotating about the polar axis at a constant angular velocity $\bar{\lambda}$ throughout the entire aerobraking pass. Under this condition, an observer fixed on the planet would not detect any winds. By adding or subtracting the rigid rotation component from the inertial zonal winds, Eq. (6) can be used to calculate the wind velocity with respect to the spacecraft.

$$
\boldsymbol{V}_{\text {flow }}=\boldsymbol{V}_{\mathrm{S} / \mathrm{C}}+\operatorname{super}\left(\boldsymbol{\lambda} \times \boldsymbol{r}_{\mathrm{S} / \mathrm{C}}\right.
$$

\section{Aerodynamic Torques}

To evaluate the right-hand side (RHS) of Eq. (4), the velocity of the wind relative to the spacecraft must be known. The velocity of the wind relative to the spacecraft is the parameterized rotation of the atmosphere using a parameter called superrotation, shown in Eq. (6). A superrotation rate of one means that the atmosphere is rotating at the same rate as the planet. A superrotation rate greater than zero but less than one means that westerly winds are blowing, but they are slower than the rotation rate of the planet. A superrotation rate greater than one means that westerly winds are being experienced that are faster than the rotation rate of the planet. If the superrotation rate is 
less than zero, the winds are easterly. The spacecraft velocity relative to the winds is transformed into a body-fixed coordinate system by premultiplying the spacecraft position vector by a rotation matrix. This transformation yields the velocity components of the wind relative to the spacecraft.

To solve the aerodynamic-torque equations, moment and force coefficients must be calculated. Multidegree interpolation over a range of atmospheric densities from free molecular flow to deep in the transition region, a range of wind components, and a range of panel deflections are used to find values for $C_{m_{x}}, C_{m_{y}}, C_{x}$, and $C_{y}$ that correspond to the known density and panel deflection at each point during the pass. These aerodynamic coefficients, each of which correspond to a different panel deflection of 0,10 , and $20 \mathrm{deg}$ [6], are referred to the assumed spacecraft center of mass.

The original aerodynamic coefficients are not calculated about the actual spacecraft center of mass. Using the initial spacecraft centerof-mass location as the origin, the center of mass of the spacecraft has to be shifted to the location in which these coefficients are valid. The shifts in the $x, y$, and $z$ directions, represented by $\Delta x, \Delta y$, and $\Delta z$, are all constants. However, the spacecraft center of mass changes as the SAM deflects, and these shifts in the $y$ and $z$ directions are represented by $\delta y$ and $\delta z$. The aerodynamic-torque equations therefore take the form shown in Eq. (7), in which $C_{m_{x}}$ and $C_{m_{y}}$ depend on the superrotation parameter.

$$
\begin{aligned}
& N_{x}=0.5 \rho V^{2} A L \frac{\left\{C_{m_{x}}+\left[(\Delta y+\delta y) C_{z}-(\Delta z+\delta z) C_{y}\right]\right\}}{L} \\
& N_{y}=0.5 \rho V^{2} A L \frac{\left\{C_{m_{y}}+\left[(\Delta z+\delta z) C_{x}-\Delta x C_{z}\right]\right\}}{L}
\end{aligned}
$$

\section{Least-Squares Solution}

The least-squares method is used in an iterative manner to solve for zonal winds over a batch of orbits. The purpose of this differential correction is to minimize the difference between the inertia-related and aerodynamic torques in Eq. (4) over batches of orbits by varying the spacecraft center-of-mass location and the superrotation rate.

To find the least-squares solution, the LHS and RHS of Eq. (4) are balanced. That is, LHS $=$ RHS $+\varepsilon$, where the parameters are chosen to minimize the length of the residual vector $\varepsilon$. A $1 \sigma$ a priori uncertainty of $0.25 \mathrm{~N} \cdot \mathrm{m}$ is associated with the torque residuals, a $1 \sigma$ a priori center-of-mass uncertainty of $0.007 \mathrm{~m}$ is applied in the $x$ and $y$ directions, and $0.05 \mathrm{~m}$ is applied in the $z$ direction. The $1 \sigma$ a priori uncertainty on superrotation is set to 1.0. To perform the leastsquares minimization, the sensitivity matrix is calculated by finite difference partials. The sensitivity matrix consists of four separate partials, which are found by taking the partial derivatives of the known terms with respect to the three center-of-mass locations and a superrotation parameter for each orbit. Upon completion of the iteration process, the superrotations that minimize the error of the least-squares equations, along with the associated uncertainties, are determined. The center of mass of the spacecraft is held constant for the batch, and the superrotation values yield the zonal winds for each orbit. Knowing the rotation rate of Mars and the spacecraft position vector at periapsis, superrotations are converted to zonal wind velocities, and superrotation uncertainties are converted to zonal wind uncertainties. A flowchart is included in Appendix A that demonstrates the processes used to calculate zonal wind velocity.

\section{Consideration of Potential Errors}

Several error sources are considered, including accelerometer errors during the aerobraking pass and accelerations due to fuel sloshing. Effects of thruster firings and changes in mass due to fuel consumption are also considered, and an analysis was performed to determine the sensitivity of heading error on wind velocity.

Accelerometer errors associated with the density measurements are negligible at altitudes within $30 \mathrm{~km}$ of periapsis] [7] and are not

๑Data available online at http://atmos.nmsu.edu/pdsd/archive/data/mgs-maccel-5-profile-v12/mgsa_0002/catalog/inst.cat [retrieved 11 July 2007]. included in the superrotation calculations. Accelerations due to fuel sloshing are not evident on MGS. Fuel settles toward the forward end of the tank near the periapsis portion of the aerobraking pass, mitigating fuel slosh during the times at which the wind calculations are made. In addition, the effects of fuel slosh during the aerobraking of the Mars Reconnaissance Orbiter are not evident below altitudes of $150 \mathrm{~km}$. Therefore, accelerations due to fuel slosh are not considered to be a significant error source in the calculation of zonal winds at periapsis altitudes, which never exceed $135 \mathrm{~km}$.

Taking into account the effects due to thruster firings would be extremely time-consuming and require thousands of reconstructed thruster $\Delta V s$ from both the navigation and attitude control teams. Therefore, data at times of the thruster firings are identified and eliminated from all calculations, and the a priori torque residual uncertainty of $0.25 \mathrm{~N} \cdot \mathrm{m}(1 \sigma)$ is chosen accordingly. Mass decrements due to fuel depletion are taken into account during the calculation of the density profiles.

Heading errors can adversely affect the density calculations through a corresponding incorrect $C_{d}$ [see Eq. (1)] and an error in $C_{m_{x}}$, and a sensitivity study was undertaken to assess the effect of this phenomenon. Attitude requirements specified that the gyro stability shall not exceed $0.07 \mathrm{deg} / \mathrm{h}(3 \sigma)$ for $24 \mathrm{~h}$ of continuous operation. A conservative 0.1 -deg heading error was used in the sensitivity study, because star calibrations were performed near apoapsis. Results indicate that a $0.1-\mathrm{deg}$ heading error results in a $0.03 \mathrm{~kg} / \mathrm{km}^{3}$ error in density, which maps into a negligible $0.5 \mathrm{~m} / \mathrm{s}$ error in zonal wind velocity.

\section{Spacecraft Geometry}

The determination of zonal winds is highly dependent on an accurate knowledge of the spacecraft geometry. The common basis used to solve Eq. (4) is the spacecraft center of mass, but components are often referenced with respect to the nozzle exit plane. Figure $\underline{6}$ shows the dimensions of each major component with respect to the center of mass of the spacecraft.

The average mass of the entire spacecraft is $757 \mathrm{~kg}$, with the SAM weighing $39.34 \mathrm{~kg}$ and the bus/SAP combination weighing an average of $717.66 \mathrm{~kg}$.

\section{Effects of Solar Array Minus Deflection}

The SAM deflected about the $x$ axis during aerobraking passes because a solar array deployment damper failed during the cruise phase of the mission, thereby causing the partial structural failure of the SAM [8]. This panel anomaly is important in the determination of zonal winds, because the panels may have different accommodation coefficients, and there is a panel bias that varies during the course of aerobraking.

\section{Effects of Different Accommodation Coefficients}

Gas-surface accommodation coefficients represent the degree to which incident molecules achieve equilibrium with the surface. Incomplete accommodation (accommodation less than one) reduces the momentum and energy of particles transferred to the surface. As part of the aerobraking operations, the SAM was rotated 180 deg to attenuate the stresses acting on the panel. Therefore, the forwardfacing surface of the SAM is glass-covered solar cells, whereas the forward-facing surface of the SAP is a composite face sheet. Glass

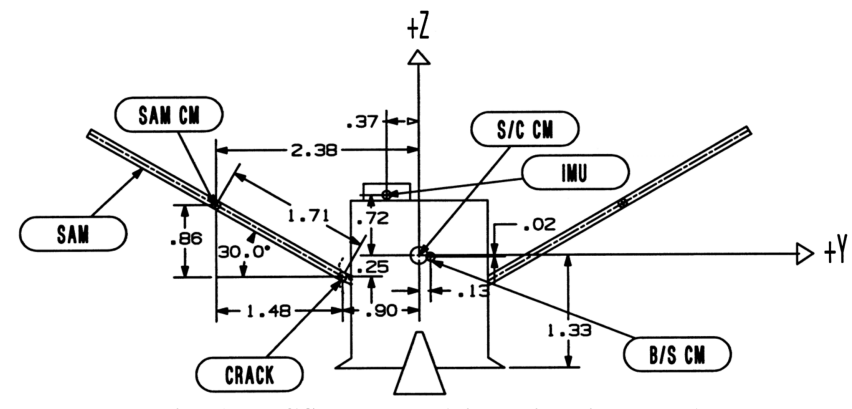

Fig. 6 MGS geometry (dimensions in meters). 
has a lower accommodation coefficient than a composite face sheet, and differences in accommodation coefficients up to 0.2 may be possible, based on limited observations of the Magellan spacecraft [6]. A lower accommodation coefficient on the SAM means that there is a greater degree of specular reflection, and forces are higher in the stream direction, which increases the aerodynamic torque. This effect causes the spacecraft to trim at yaw angles other than zero, which was assumed in the calculation of the zonal winds. Even though the trimmed yaw angle is less than $1 \mathrm{deg}$ for typical periapsis densities, the spacecraft was rolled $180 \mathrm{deg}$ about the $y$ axis near P138. Subsequently, MGS assumed a different equilibrium yaw angle with respect to the planet, whereas the zonal winds were still the same. Because zonal wind velocity is a function of yaw angle, discontinuities in the results exist near the orbit in which the roll was executed. Because there is no way of accurately determining the yaw angle bias induced by the differential accommodation coefficients during each pass, it is considered to be an acceptable, albeit uncorrectable, source of error.

\section{Effect of Panel Bias}

The zonal wind velocities are highly dependent on an accurate knowledge of the spacecraft center-of-mass location. As the SAM deflects, the center of mass of the spacecraft is shifted in both the $y$ and $z$ directions, as shown in Eq. ( $)$ :

$$
\Delta C M_{\mathrm{S} / \mathrm{C}}=\frac{\left(M_{\mathrm{SAM}} \Delta C M_{\mathrm{SAM}}\right)}{M_{\mathrm{S} / \mathrm{C}}}
$$

Figure 7 shows the change of the spacecraft center of mass in the $y$ and $z$ directions as a function of the SAM deflection.

A change in spacecraft center of mass due to the SAM deflection results in a corresponding change in $C m_{x}$, which must be accurately calculated to solve for zonal wind velocity. $C m_{x}$ varies as the SAM deflection changes according to Eqs. (9) and (10).

The changes in $C m_{x}$ due to the SAMM deflection are shown in Fig. 3. $C m_{x}$ has a negative slope, indicating positive stability of the spacecraft. It also has an equilibrium position at a yaw angle of $0 \mathrm{deg}$ with no SAM deflection. With a SAM deflection of $10 \mathrm{deg}$, a yaw angle of 5 deg would be necessary for the spacecraft to maintain a state of equilibrium, resulting in $C_{m_{x}}$ shifting from 0 to -0.01 . Therefore, the effects of panel position on the spacecraft center of mass are not negligible when calculating zonal wind velocity.

The SAM experienced a bias during both mission phases. The effects of panel deflection about $x$ are taken into account during calculation of zonal wind velocity using a panel-deflection model, assuming the SAM is joined to the bus with a nonlinear torsional spring. However, a drifting bias occurred before and after periapsis in both phases, with the largest effect seen in phase 1.

$$
C_{m_{x}}=C_{m_{x 0}}+\frac{\left(\delta y_{\mathrm{SAM}} \cdot C_{z}-\delta z_{\mathrm{SAM}} \cdot C_{y}\right)}{L}
$$

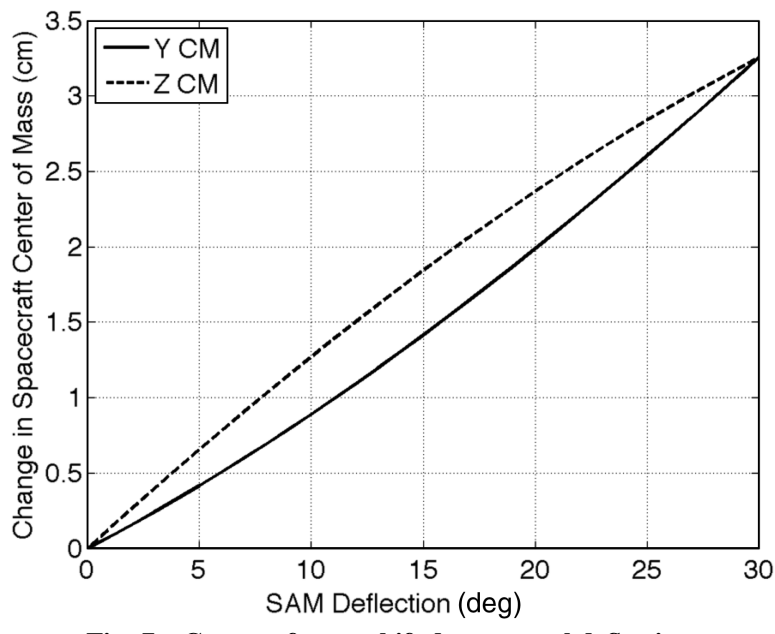

Fig. 7 Center-of-mass shift due to panel deflection.

$$
C_{m_{y}}=C_{m_{y 0}}+\frac{\left(\delta z_{\mathrm{SAM}} \cdot C_{x}-\delta x_{\mathrm{SAM}} \cdot C_{z}\right)}{L}
$$

Because zonal wind velocity is highly dependent on the spacecraft center-of-mass location, which is dependent on the SAM position, this bias cannot be ignored. The bias reached its maximum value of $2.1 \mathrm{deg}$ on orbit 152 . A large discontinuity occurs near orbit 80 , in which the bias actually changes direction. The cause of this sudden change is not known, but one theory attributed this change to the 0.16-Hz mode being strongly excited on some orbits, which caused the cracked face sheets to suddenly slip past each other. Discontinuities in the zonal wind model are reduced by determining the average panel bias at the periapsis of each orbit and adjusting the spacecraft center of mass to account for the center-of-mass offset that results from such a deflection. The bias is taken into account by fitting a curve through the inbound and outbound measured paneldeflection data. The average cubic polynomial between the inbound and outbound is then calculated, and this model is assumed to be the panel bias near periapsis, the region in which zonal wind velocities are calculated. A bias discontinuity also exists between the end of phase 1 and the beginning of phase 2 , and it is postulated that this change is induced by the numerous attitude changes that occurred during the science phasing orbits.

Figure 8 a shows the inbound, outbound, and average panel biases for phase $\overline{1}$, and Fig. $8 \mathrm{~b}$ shows the inbound, outbound, and average panel biases for phase $\overline{2}$ up through P992. Subsequent orbits are not used to calculate zonal winds due to the low orbital eccentricity, which results in a large latitudinal change during the aerobraking passes. Zonal winds are assumed to be constant throughout the entire pass and are meaningless over a wide array of latitudes.

\section{Results}

For each aerobraking pass, the $x$ torques and $y$ torques are calculated from the left-hand and right-hand sides of Eq. (4), while taking into account the SAM bias. Confidence in the model is gained when plots of the external and inertia-related torques compare well. For most orbits, both the $x$ torques and $y$ torques have good correlation between the left and right-hand sides of Eq. (4), but if the correlation coefficient falls below 0.9 , the winds from that orbit are not calculated.

Generally, the aerodynamic torques are less than the inertiarelated torques, but the deviations between the known torques and aerodynamic torques are small enough to make the potential error in zonal wind calculations acceptable $(\sim 5 \mathrm{~m} / \mathrm{s} 1 \sigma)$.

The least-squares process is run in batches of approximately ten orbits each. For one orbit, the center-of-mass location and the superrotation are weekly separated, but for batches of approximately 10 or more orbits, enough variation in the superrotation exists to make the separation possible. The superrotation rate is calculated for each orbit, whereas the spacecraft center of mass is held constant for all orbits in the batch. The zonal wind velocity at periapsis is calculated using Eq. (ㅁ).

\section{Phase-1 Winds}

Figure 9 shows that the phase- 1 zonal wind magnitude at periapsis reaches a high value of approximately $300 \mathrm{~m} / \mathrm{s}$ during P53. This high velocity was reached at the same time that a large dust storm erupted in the Noachis Terra region of Mars in November 1997. P53 occurred during the southern spring season, a season that usually marks the onset of maximum dust-storm activity. In general, the phase- 1 zonal winds are observed to be westerly (west-to-east flow) at the periapsis altitude of approximately $120-130 \mathrm{~km}$. Figure 10 shows the evolution of periapsis altitude throughout phase- 1 orbits of interest.

This wind speed experiences a downward trend as latitude varies in a nearly linear fashion from $37-61^{\circ} \mathrm{N}$ over P40-P201. Immediately before the onset (before P49) and after the Noachis storm decay (beyond P90), zonal wind magnitudes are approximately $75-100 \pm 3 \mathrm{~m} / \mathrm{s}$ ( $1 \sigma$, westerly). Maximum dust- 


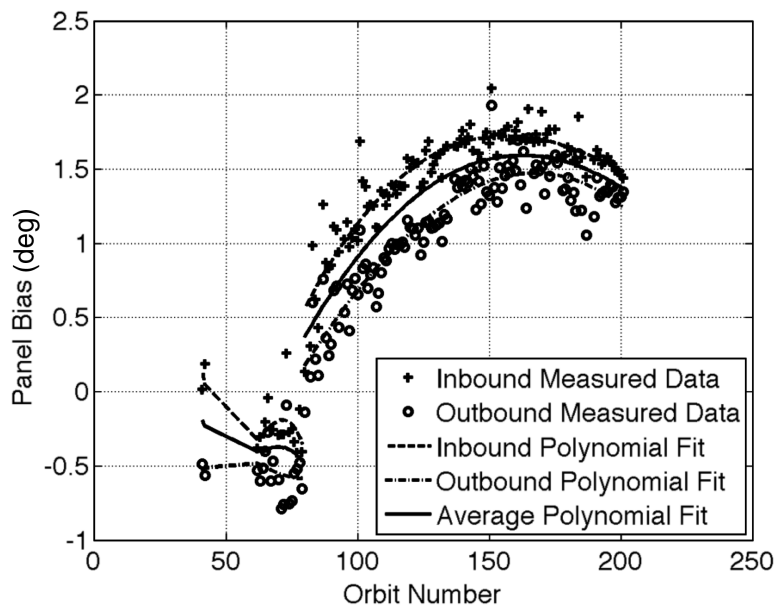

a) Phase 1

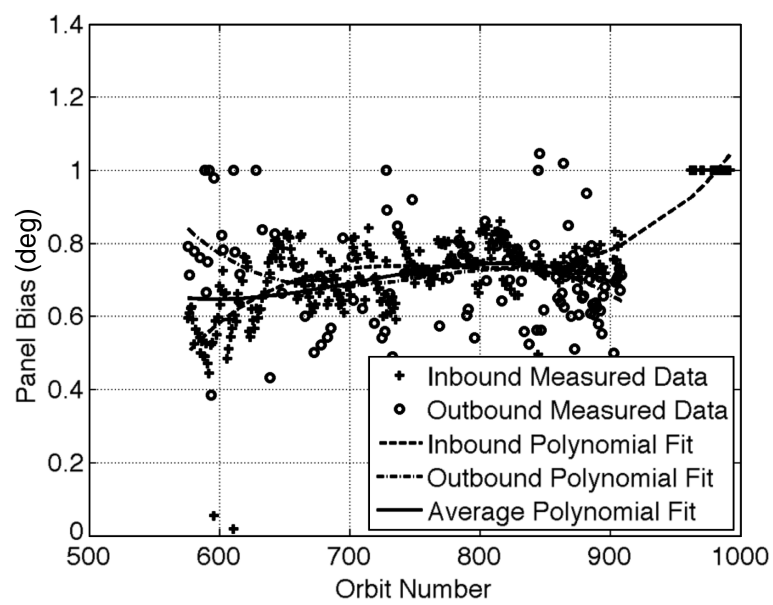

b) Phase 2

Fig. 8 Panel bias and polynomial fits.

storm winds (near P53) are approximately $\sim 200 \mathrm{~m} / \mathrm{s}$ stronger. Over the higher-latitude regions (beyond P100), the zonal wind magnitudes decrease significantly and, in some instances, are noticed to be easterly (east-to-west flow).

When MGS was at periapsis P40, the season was southern spring (solar longitude $L_{S}=215^{\circ}$ ), but by orbit 201, the southern summer season $\left(L_{S} \sim 300^{\circ}\right)$ had arrived. The northern-hemisphere $\left(37-61^{\circ} \mathrm{N}\right)$ wind velocities tend to decrease from southern spring to summer (northern fall to winter). Superimposed on this plot of observed zonal wind magnitudes are simulated mean and $\pm 3 \sigma$ curves for zonal wind magnitudes obtained from the MARSGRAM2005 (MG2005) empirical model [9]. These simulated winds are provided for the periapsis location (latitude, longitude, solar local time, and altitude), seasonal conditions ( $\mathrm{L}_{S}$ and dust opacities), and solar fluxes encountered during MGS phase-1 aerobraking for orbits P40-P201. An average integrated visible dust opacity of $\sim 1.0$ is assumed throughout these phase- 1 orbits, in accordance with observed opacities outside the Noachis dust-storm period ( $\sim$ P49-P90). It is clear that MG2005 simulations capture the basic features of the zonal wind trends with latitude and season during this interval, except during the Noachis dust-storm onset and decay periods. During this time, observed zonal winds are much stronger (up to $\sim 300 \mathrm{~m} / \mathrm{s}$ ). The MG2005 model is constructed from coupled model simulations of the NASA Ames Research Center's Mars global circulation model (MGCM) and the National Center for Atmospheric Research at the University of Michigan (NCAR/Michigan) Mars thermospheric general-circulation model (MTGCM) for specific Mars seasonal, dust, and solar conditions $[\underline{9}, \underline{10}]$.

A comparison of the calculated zonal winds and simulated MG2005 winds using a simplified dust-storm-evolution scheme was also made. However, the dust-storm effects were not wellrepresented, because of limitations of the dust-storm-evolution

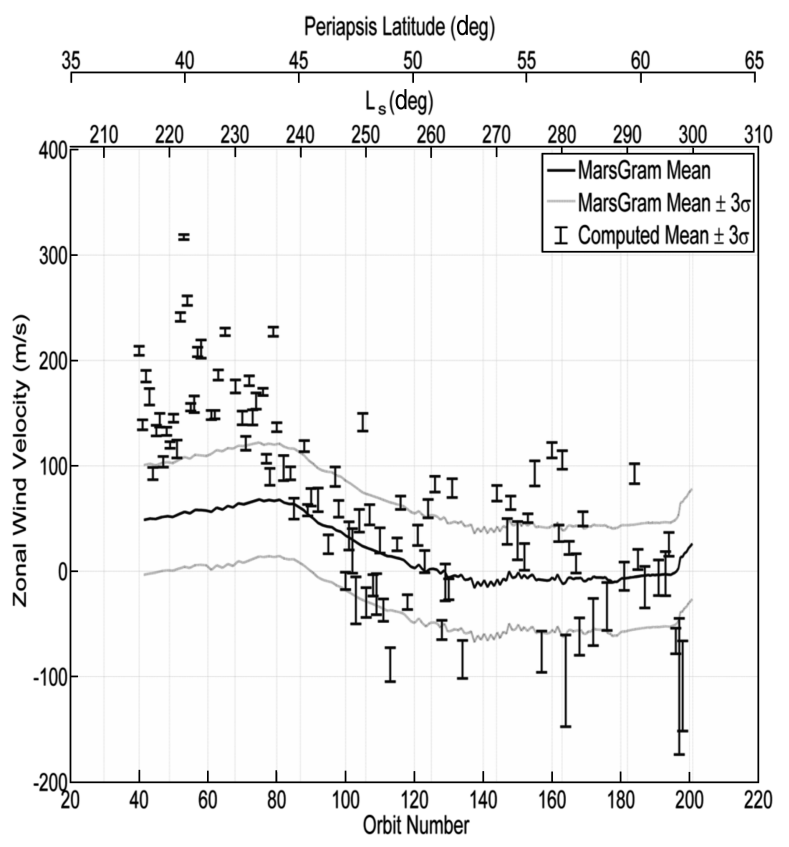

Fig. 9 Computed phase-1 zonal winds with 3- $\sigma$ error bars superimposed on the mean and $\pm 3-\sigma$ winds computed from MARSGRAM 2005.

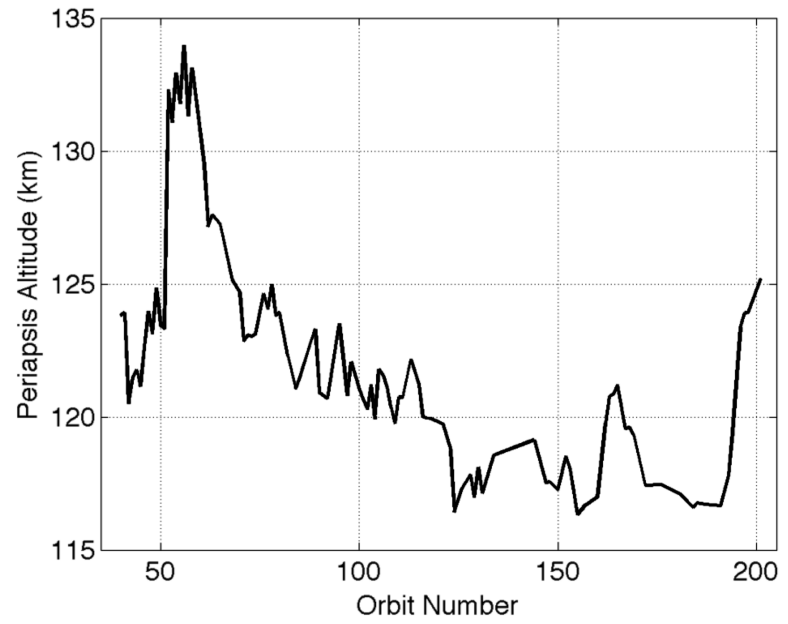

Fig. 10 Periapsis altitude of phase-1 orbits of interest.

model. The output did not produce a significantly different profile than that shown in Fig. 9 , but the derived winds in Fig. 9 provide a strong constraint for a comprehensive evolving-dust-storm simulation using the coupled MGCM-MTGCM codes.

\section{Phase-2 Winds}

The phase- 2 zonal wind velocities were calculated using the same methods as in phase 1 . It was recognized that phase- 2 orbits occurred during the MGS aerobraking period when orbit periods were less than $12.5 \mathrm{~h}$, and the spacecraft was in the atmosphere (below $200 \mathrm{~km}$ ) for more than $500 \mathrm{~s}$ on each aerobraking pass. This long residence time in the atmosphere required many spacecraft thruster firings (more than phase 1), for which numerous individual corrections to model each thruster firing were needed before useful wind speeds could be derived. These corrections were not applied in this exercise. However, this concern may be mitigated by reducing the length of the data arc and focusing only on those orbits with the fewest number of thruster firings. Results of this exercise will be addressed in a future paper.

\section{Seasonal Effects}

During phase 1, zonal winds are highest during southern spring (local northern fall) and tend to decrease as the southern summer 
season progressed. Phase-1 westerly zonal winds are consistent with northern-hemisphere $\left(37-60^{\circ} \mathrm{N}\right)$ temperatures that are generally decreasing with increasing latitude. In addition, excessively high winds are most likely to occur when dust storms erupt, which is common in the southern spring season.

\section{Conclusions}

The primary objective of Mars Global Surveyor aerobraking operations was to reduce the orbital period and eccentricity. However, data taken during aerobraking operations have proven useful in determining zonal wind patterns. The results of the research documented in this report show that winds during phase 1 of aerobraking are primarily westerly. However, on occasion, wind patterns are shown to be easterly. The easterly winds occurred only at latitudes between $\sim 45$ and $60^{\circ}$. During a regional dust storm, which began at about orbit 49 and lasted for a number of weeks, extremely high westerly winds (up to $300 \mathrm{~m} / \mathrm{s}$ ) occurred suddenly, rising more than $100 \mathrm{~m} / \mathrm{s}$ above the normal wind velocity.

Noticeable and significant seasonal effects are observed. The latitude of wind sampling must be considered when describing seasonal effects. For example, westerly zonal winds are strongest for the northern hemisphere $\left(37-60^{\circ} \mathrm{N}\right)$ in late fall, when temperatures are generally decreasing with increasing latitude. Southern hemisphere westerly zonal winds are strongest during local southern winter, when temperatures are generally decreasing toward the local winter pole. Furthermore, excessively high winds are most likely to occur when dust storms erupt, a common occurrence in the southern spring season.

Future efforts include calculation of mean zonal winds at constant reference altitudes using MGS, Odyssey, and Mars Reconnaissance Orbiter data and comparing the results with MG2005 predictions. Such calculations will provide insight into zonal wind vertical profiles, including wind shears, at altitudes of 100-140 km. Latitudinal and longitudinal variations at constant altitudes will also be analyzed. The derived zonal winds presented in Fig. 9 provide strong constraints for calculated zonal winds resulting from a comprehensive evolving-dust-storm simulation using the coupled MGCM-MTGCM codes. The radiative, dynamic, and dust-transport feedbacks operating in the Mars atmosphere during the Noachis storm, giving rise to these zonal wind variations, can then be investigated in detail.

\section{Appendix A: Flow Chart to Solve for Zonal Winds}

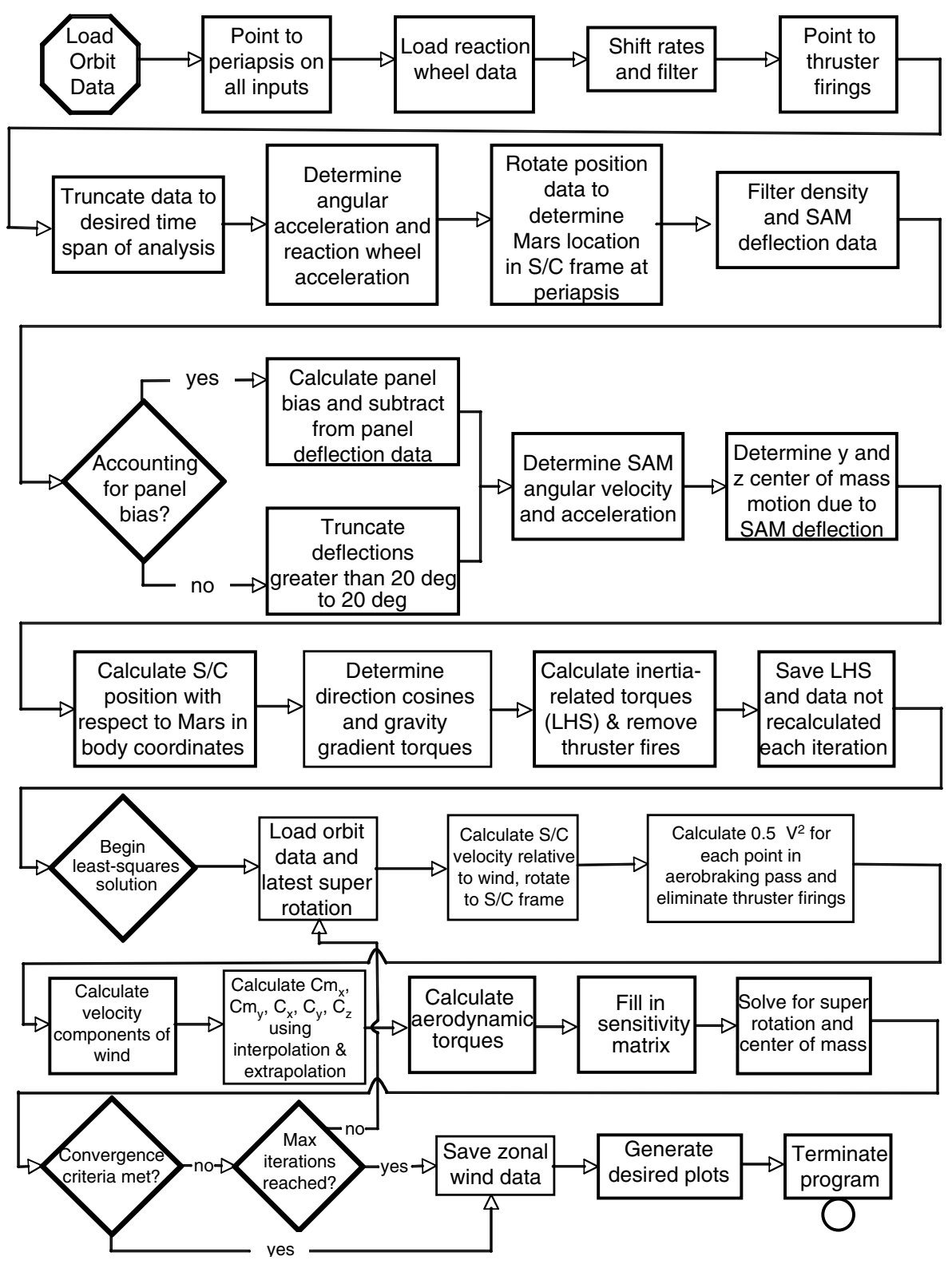




\section{Acknowledgments}

The work described in this paper was started at the George Washington University, Joint Institute for Advancement of Flight Sciences, sponsored by NASA, and was completed at the Jet Propulsion Laboratory, California Institute of Technology, under a contract with NASA. NASA Mars Data Analysis Program support for S. Bougher is recognized, and Mars Global Surveyor project support is appreciated.

\section{References}

[1] Dallas, S. Sam, "Mars Global Surveyor Mission," IEEE Aerospace Conference, Vol. 4, Inst. of Electrical and Electronics Engineers, Piscataway, NJ, 1997, pp. 173-189.

[2] Tolson, R. H., Keating, G. M., Cancro, G. J., Parker, J. S., Noll, S. N., and Wilkerson, B. L., "Application of Accelerometer Data to Mars Global Surveyor Aerobraking Operations," Journal of Spacecraft and Rockets, Vol. 36, No. 3, May-June 1999, pp. 323-329.

[3] Keating, G. M., Bougher, S. W., Zurek, R. W., Tolson, R. H., Cancro, G. J., Noll, S. N., Parker, J. S., Schellenberg, T. J., Shane, R. W., Wilkerson, B. L., Murphy, J. R., Hollingsworth, J. L., Haberle, R. M., Joshi, M., Pearl, J. C., Conrath, B. J., Smith, M. D., Clancy, R. T., Blanchard, R. C., Wilmoth, R. G., Rault, D. F., Martin, T. Z., Lyons, D. T., Esposito, P. B., Johnston, M. D., Whetzel, C. W., Justus, C. G., and Babicke, J. M., "The Structure of the Upper Atmosphere of Mars: InSitu Accelerometer Measurements from the Mars Global Surveyor," Science, Vol. 279, Mar. 1998, pp. 1672-1676.

[4] Cancro, G. J., Tolson, R. H., and Keating, G. M., "Operational Data
Reduction Procedure for Determining Density and Vertical Structure of the Martian Upper Atmosphere from Mars Global Surveyor Accelerometer Measurements," NASA CR-1998-208721, 1998, pp. 37-38.

[5] Meirovitch, L., Methods of Analytical Dynamics, McGraw-Hill, New York, 1970, p. 437.

[6] Wilmoth, R. G., Rault, D. F., Cheatwood, F. M., Engelund, W. C., and Shane, R. W., "Rarefied Aerothermodynamic Predictions for Mars Global Surveyor," Journal of Spacecraft and Rockets, Vol. 36, No. 3, May-June 1999, pp. 314-322.

[7] "PDS Atmospheres Data Set Catalog," PDS Atmospheres Node [online database], http://pds-atmospheres.nmsu.edu/cgi-bin/getdir.pl?dir=data\&volume=mgsa_0002 [retrieved 11 July 2007].

[8] Lyons, Daniel T., Beerer, J. G., Esposito, P., Johnston, M. D., and Willcockson, W. H., "Mars Global Surveyor: Aerobraking Mission Overview," Journal of Spacecraft and Rockets, Vol. 36, No. 3, MayJune 1999, pp. 307-313.

[9] Justh, H. L., Justus, C. G., and Keller, V. W., "Global Reference Atmospheric Models, Including Thermospheres, for Mars, Venus, and Earth," AIAA Paper 2006-6394, Aug. 2006.

[10] Bougher, S. W., Murphy, J. R., Bell, J. M., Lopez-Valverde, M. A., and Withers, P. G., "Polar Warming in the Mars Lower Thermosphere: Seasonal Variations Owing to Changing Insolation and Dust Distributions," Geophysical Research Letters, Vol. 33, No. 2, 2006, Paper L02203.
A. Ketsdever Associate Editor 